\title{
The band-gap structures and recovery rules of generalized $n$-component Fibonacci piezoelectric superlattices
}

\author{
Da Liu and Weiyi Zhang \\ Nanjing National Laboratory of Microstructures and Department of Physics, Nanjing \\ University, Nanjing 210093, China
}

\begin{abstract}
In this communication, the band-gap structures of $n$-CF piezoelectric superlattices have been calculated using the transfer-matrix-method, the self-similarity behavior and recovery rule have been systematically analyzed. Consistent with the rigorous mathematical proof by $\mathrm{Hu}$ et al.[A. Hu et al. Phys. Rev. B. 48, 829 (1993)], we find that the $n$-CF sequences with $2 \leq n \leq 4$ are identified as quasiperiodic. The imaginary wave numbers are characterized by the selfsimilar spectrum, their major peaks can all be properly indexed. In addition, we find that the $n=5$ sequence belongs to a critical case which lies at the border between quasiperiodic to non-quasiperiodic structures. The frequency range of self-similarity pattern approaches to zero and a unique indexing of imaginary wave numbers becomes impossible. Our study offers the information on the critical 5-CF superlattice which was not available before. The classification of band-gap structures and the scaling laws around fixed points are also given.
\end{abstract}

Keywords: Polaritons, Piezoelectric films, Wave transmission.

\section{INTRODUCTION}

From a symmetrical point of view, the solids in nature are classified as crystals, quasicrystals, and noncrystals [1]. The crystal structure is characterized by a periodic arrangement of atoms in space, the waves propagating in crystals have a Bloch wave form. According to the Bloch theorem all states are extended in space and eigenenergies form continuous band structures. For fully disordered noncrystal, it is believed that all states are localized in space and energy spectra are singular continuous. Quasicrystal is a unique type of structure which lies at the boundary between translation invariant crystals and random glassy materials [2]. Though lacking long-range translational symmetry, it possesses a certain orientational order. To understand the interim situation between crystals and noncrystals, much research works have been carried out on quasicrystals, especially since the experimental discovery of quasicrystal phase in an Al-Mn alloy with icosahedral symmetry [3]. The simplest structural model 
describing quasicrystals is the Fibonacci lattice model. It has received a great

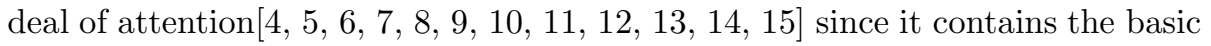
ingredients of quasicrystals and is relatively easy to deal with. The Fibonacci lattice model is famous for its Cantor-set spectrum, the self-similar spectrum is identified as critical eigenstates which is neither extended as in periodic system, nor localized as in disordered ones [2].

To explore the spectral evolution from the translational invariant crystals to fully disordered materials, a structural model which is tunable between the two extreme cases are highly desirable. A simple Fibonacci lattice model is certainly a good candidate since its symmetry lies in between, but it is not good enough since its order is not adjustable. In this respect, the generalized Fibonacci lattice model with $n$-component $(n$-CF) offers an excellent choice and its order can be tuned by the parameter $n[16]$. The $n$-CF lattice can be obtained by repeated application of the concurrent substitution rules $T A_{1}=A_{1} A_{n}, T A_{i}=$ $A_{i-1}(i=2, \ldots, n)$, where $A_{1}, A_{2}, \ldots, A_{n}$ are the $n$-components and $T$ is the substitution operator. The $l$ th generation sequence of $n$-CF lattice is defined as $S_{l}=T^{l} A_{1}$ and the generalized $n$-CF lattice corresponds to $S_{\infty}$. The first few generation sequences are given by $S_{0}=A_{1}, S_{1}=A_{1} A_{n}, S_{2}=A_{1} A_{n} A_{n-1}$, $\ldots$, and $S_{n}=A_{1} A_{n} A_{n-1} \ldots A_{3} A_{2} A_{1}$. In general, $S_{l}=S_{l-1}+S_{l-n}(l \geq n)$. The generalized $n$-CF lattice recovers the periodic lattice if $n$ is set to $n=1$, it also naturally reproduces the standard Fibonacci lattice when $n=2$. What's unique about the generalized $n$-CF lattice is that it can be tuned smoothly from periodic, to quasiperiodic, and then finally to non-quasiperiodic lattices as $n$ changes. As shown by $\mathrm{Hu}$ et al. [16], the information on the quasiperiodicity of a $n$-CF lattice is encoded in the substitution matrix

$$
T=\left(\begin{array}{ccccc}
1 & 0 & 0 & \ldots & 1 \\
1 & 0 & 0 & \ldots & 0 \\
0 & 1 & 0 & \ldots & 0 \\
\vdots & \ddots & \ddots & \ddots & \vdots \\
0 & \ldots & 0 & 1 & 0
\end{array}\right)_{n \times n}
$$

whether the $n$-CF lattice is quasiperiodic or not is closely associated with the Pisot number of characteristic polynomial equation $\lambda^{n}-\lambda^{n-1}-1=0$. A Pisot number is present if the characteristic polynomial equation has only one root $1<\lambda_{0}^{(n)}<2$ and all its conjugate roots have their norms less than 1. Following the Bombier-Taylor theorem, the existence of Pisot number guarantees that the $n$-CF lattice is quasiperiodic and can be generated by the cut and projection method [17]. Hu et al. [16] showed that quasiperiodic lattice exists only if $2 \leq$ $n \leq 5 ; n=1$ corresponds to a periodic lattice while lattices with $n>5$, though still ordered, is no longer quasiperiodic. In fact, a close inspection of the root structure suggests that $n=5$ lattice should be classified as a critical case since there is a pair of conjugate roots having their norms equal 1, which neither belongs to the quasiperiodic case where all conjugate roots have their norms less than 1 , nor belongs to a non-quasiperiodic case where some of the conjugate roots have their norms larger than 1 . The Pisot numbers are $\lambda_{0}^{(2)}=1.61803$, 
$\lambda_{0}^{(3)}=1.46557, \lambda_{0}^{(4)}=1.38028$, and $\lambda_{0}^{(5)}=1.32472$ for $n=2,3,4,5$, respectively.

Above discussion suggests that $n$-CF lattice covers a wide range of lattice types from periodic, quasiperiodic, critical, and non-quasiperiodic lattices, it offers a natural platform to study the spectra evolution with structural ordering. Though extensive investigations have been carried out on the electronic [4, 5, 6, 6, 8] , vibrational [9, 10, 11], and dielectric [12, 13, 14, 15] properties of quasiperiodic structures as a vehicle to study the evolution process from ordered periodic structures to disordered solids, the most previous studies concentrated on the standard Fibonacci lattices [4, 5, 6, 7, 8, 9, 10, 11, 12, 13, 14, 15] and the generalized 2-component Fibonacci lattices 18, 19, 20, 21, 22, 23, 24], the generalized $n$-CF lattices are mostly discussed in the context of structural properties 25]. Furthermore, the previous studies mostly dealt with the single degree problems, and studies on the mode-coupling problems with energy transfer between different degrees of freedom are only a few [26, 27, 28]. Thus, we have carried out a comprehensive study in this communication on the band-gap structures of $n$-CF piezoelectric superlattices. The transfer-matrix-method is explored so that the band-gap structures can be most easily visualized through the imaginary wave number. Our study show that the band-gap structures can be classified into a series of big cluster in the frequency spectrum. For each $n$ CF superlattice, the self-similar spectrum and recovery rule are present within each generation and across different generations $l$. Consistent with the rigorous mathematical proof, we find that the $n$ - $\mathrm{CF}$ superlattices with $2 \leq n \leq 4$ can be identified as quasiperiodic. The band-gaps are characterized by the self-similarity behavior and all the gaps can be properly indexed; The $n=5$ superlattice belongs to a critical case which lies at the border between quasiperiodic to non-quasiperiodic structures. The frequency range of self-similar spectrum approaches zero, a unique indexing of band-gaps becomes impossible.

\section{MODEL}

In this communication, we use superlattices made of piezoelectric $\mathrm{LiNbO}_{3}$ compound as our model systems [26]. To distinguish different components of $n$-CF superlattices 16], each component is made of a pair of positively and negatively polarized ferroelectric domains of varying lengths $\left(L_{+}^{i}, L_{-}^{i}\right)$. For simplicity and also for setting up length scale, the lengths of positively polarized domains of all components are taken to be the same $L_{+}$, while the lengths of negatively polarized domains are used to differentiate the different components. A typical superlattice for a given $n$-CF sequence and for a given generation $l$ is illustrated in Fig. 1. The experimental setting and material parameters are described in detail in the references. 27, 28]

To calculate the band-gap structures of $n$-CF lattices, we use the sequence of $l$ th generation as a supercell to form a periodic lattice. In this case, the lattice constant $a_{l}=\sum_{i=1}^{n} F_{l}^{i} \bar{L}^{i}$ where $F_{l}^{i}$ is the number of $i$ th component in $l$ th generation sequence and $\bar{L}^{i}=\pi L^{i} / L_{+}$is the reduced length of $i$ th component [27]. The corresponding reciprocal lattice vectors are $K_{m_{l}}=\left(2 \pi / a_{l}\right) m_{l} \mathrm{~s}$ with $m_{l}$ 
denoting an integer, the reduced wave number $-\pi / a_{l}<\bar{k} \leq+\pi / a_{l}$. Thus, the essential information on the $l$ th generation of $n$-CF sequence is included in its Fourier components $\theta\left(m_{l}\right)$. The band-gap opens at the reduced frequency $\bar{\omega} \approx K_{m_{l}}$ if $\theta\left(m_{l}\right) \neq 0$. For the special case where $\bar{L}_{+}^{i} \equiv \pi$ and $\bar{L}_{-}^{i} / \pi$ can all be written as rational numbers, $\bar{L}^{i}=\pi\left[1+b_{-}^{i} / a_{-}^{i}\right]$ with the greatest common $\operatorname{divisor} \operatorname{gcd}\left(a_{-}^{i}, b_{-}^{i}\right)=1$, the reciprocal lattice vectors can also be written as rational numbers

$$
K_{m_{l}}=\frac{2 m_{l} \times \operatorname{lcm}\left(a_{-}^{1}, a_{-}^{2}, \cdots, a_{-}^{n}\right)}{\operatorname{lcm}\left(a_{-}^{1}, a_{-}^{2}, \cdots, a_{-}^{n}\right) \cdot \sum_{i}^{n} F_{l}^{i}\left(1+b_{-}^{i} / a_{-}^{i}\right)}
$$

with $\operatorname{lcm}\left(a_{-}^{1}, a_{-}^{2}, \cdots, a_{-}^{n}\right)$ denoting the least common multiple. It is easy to shown that $\theta\left(m_{l}\right)$ vanishes when $m_{l}=$ integer $\times \operatorname{lcm}\left(a_{-}^{1}, a_{-}^{2}, \cdots, a_{-}^{n}\right) \cdot \sum_{i}^{n} F_{l}^{i}(1+$ $\left.b_{-}^{i} / a_{-}^{i}\right)$. In this case, the band-gap structures are divided into big clusters, the self-similarity and scaling behavior in each cluster can be used to check whether the given superlattice is quasiperiodic or not.

\section{NUMERICAL RESULTS AND DISCUSSIONS}

To study the spectral evolution with generation $l$ for $n$-CF superlattices, usually the furcation pattern of eigenmodes is traced to analyze the self-similarity property and to search for the fixed point. When system involves continuum eigenmode spectra, it is better to track the furcation pattern of band-gap instead of the eignemodes since the discrete band-gap structures encode the essential information of $n$-CF sequences. As the band-gap represents the forbidden frequencies of eigenmodes, the wave propagating in this frequency range is attenuated at an imaginary wave number $\operatorname{Im} \bar{k}$. For polaritons which involve the coupling between electromagnetic wave and acoustic wave, the effective dielectric function in the vicinity of a band-gap always diverges at low band-gap edge while continuously approaches to zero at high band-gap edge. This suggests that the asymmetrical peaks symbolizing imaginary wave numbers can be used to visualize the band-gap structures. In the following, we shall systematically analyze the band-gap spectra of $n$-CF superlattices as functions of generation $l$ and component $n$.

Since the 2-CF sequence corresponds to the standard Fibonacci lattices which have been extensively studied before 28], we concentrate on $3 \leq n \leq 5$ cases below. In choosing the components of $n$-CF sequence, we follow the rule so that $\bar{L}^{1}>\bar{L}^{n}>\bar{L}^{n-1} \cdots>\bar{L}^{2}[16]$. In Fig. 2, the imaginary wave numbers $\operatorname{Im} \bar{k}$ as functions of frequency $\bar{\omega}$ are presented for the 9th, 12th, and 16th generations of 3-CF superlattices. The three components are chosen as $\bar{L}_{-}^{1}=\pi$, $\bar{L}_{-}^{2}=1 \pi / 3$, and $\bar{L}_{-}^{3}=2 \pi / 3$. The overall patterns of the band-gap spectra look quite similar among different generations and the positions of major bandgaps are essentially independent of generations. Within the same big cluster, the spectra demonstrate a certain symmetry with respect to the center of big cluster $(\bar{\omega}=3)$. In addition, the big cluster is further subdivided into three smaller clusters and each subcluster has its spectral center. The spectral detail 
increases with generation $l$ for a given $n$, thus the self-similarity pattern and scaling law can be analyzed when $l$ is large enough.

To check the quasiperiodicity of 3-CF superlattice, we follow Hu et al.'s procedure [16] and identify the major peaks of $n$-CF superlattice. According to the cut and projection method[17], the reciprocal lattice vectors are given by

$$
\bar{k}\left[h_{1}, h_{2}, \cdots, h_{n}\right]=2 D^{-1} \sum_{i=1}^{n} h_{i} \eta_{i},
$$

where $D=\sum_{i=1}^{n} \bar{L}^{i} \eta_{i}$ is an average lattice constant and $h_{i}$ s are any integers. $\eta_{i}$ is the relative weight of $i$ th component and satisfies $1 / \eta_{n}=\eta_{n} / \eta_{n-1}=$ $\cdots=\eta_{3} / \eta_{2}=\lambda_{0}^{(n)}$, and $\lambda_{0}^{(n)}$ is the Pisot number of substitution matrix of $n$-CF sequence. For quasiperiodic superlattices of $n$-CF type $(2 \leq n \leq 5), D$ converges quickly for large generation $l$. $D$ does not converge for aperiodic superlattices when $n>5$. Since the band-gap takes place approximately at $\bar{\omega} \approx K_{m_{l}}$, one can properly identify the various major peaks by using $K_{m_{l}}=\bar{k}\left[h_{1}, h_{2}, \cdots, h_{n}\right]$. In Fig. 2c, the major peaks of 3-CF superlattice of large generation are labeled in this way. To investigate the self-similarity property of 3-CF superlattices, the spectra of imaginary wave numbers are presented in Fig. 3 for the 9th and 16th generations. With regarding to both the positions and magnitudes of the peaks, there is a clear one-to-one correspondence between them in the vicinity of the cluster center. The frequency range of the self-similar patterns is quite broad extending from $\bar{\omega}=2.29$ to $\bar{\omega}=3.71$. The dynamical property of 3-CF superlattices is recovered after 7 -generation and the scaling parameter is $\left(\lambda_{0}^{(3)}\right)^{7}$.

Since the peak labeling of band-gaps is one of the essential feature of quasiperiodic superlattices, it is of interest to see how the situation goes for the $4-\mathrm{CF}$ and 5 - $\mathrm{CF}$ superlattices. In Fig. $4 \mathrm{a}$, the spectrum for 12 th generation of 4 -CF superlattice is presented, the four components are $\bar{L}_{-}^{1}=4 \pi / 3$, $\bar{L}_{-}^{2}=1 \pi / 3, \bar{L}_{-}^{3}=2 \pi / 3, \bar{L}_{-}^{4}=3 \pi / 3$. While Fig. $4 \mathrm{~b}$ is the similar spectrum for the 12 th generation of 5 -CF superlattice with component setting $\bar{L}_{-}^{1}=6 \pi / 3$, $\bar{L}_{-}^{2}=1 \pi / 3, \bar{L}_{-}^{3}=2 \pi / 3, \bar{L}_{-}^{4}=4 \pi / 3, \bar{L}_{-}^{5}=5 \pi / 3$. Labeling the band-gap peaks is a very tedious job for multi-component Fibonacci superlattices since more component means denser distribution of reciprocal lattice vectors. For the 4CF superlattice, we are able to identify the major band-gap peaks with easy. As shown in Fig. 4a, all major peaks are uniquely labeled as it should be for the quasiperiodic lattice. The situation becomes more complex for 5-CF superlattice since it lies at the border between quasiperiodic and non-quasiperiodic structures. We find that there exists three or more possible labelings for some major peaks, and their difference in wave number is less than $10^{-15}$, smaller than the effective digits that double precision number can offer. This non-uniqueness seems peculiar to 5 -CF superlattices, we believe that it may be related to its critical nature of the structure.

Unlike the 3-CF superlattices whose band-gap spectra recovers every 7 th generation around the fixed point at the cluster center, we find that the spectra for 4 -CF superlattices recovers every 15 th generation. Fig. 5 compares the imaginary wave number spectra for both the 12 th generation and expanded view of 
27 th generation scaled by $\left(\lambda_{0}^{(4)}\right)^{15}$. Due to one more component in composing the superlattice, the frequency range of self-similar patterns is significantly reduced in comparison with that of 3 - $\mathrm{CF}$ superlattices. But a clear one-to-one correspondence is still evident in the narrow range $2.865<\bar{\omega}<3.135$ both for peak positions and magnitudes. If we summarize the recovery rule get so far for the fixed point near the cluster center, following deductive formula seems reasonable $\Delta l=2^{n}-1$. It predicts that the spectra recover every $\Delta l=1,3,7,15$ for the periodic, standard Fibonacci [28], 3-CF, and 4-CF superlattices. Of course, the above statement holds only for the fixed point near the spectral cluster center. As a matter of fact, there are many fixed points for a given dynamical system [29, 30], and they do not share the same scaling parameter at all.

If the deductive formula is right, one would expect to find the band-gap spectra of 5-CF superlattices recover every 31st generation, but we did not succeed. Though for different settings of components we are able to find an approximate fixed point around $\bar{\omega}=3$ for the $3 \mathrm{rd}$, 7 th, and 21 st generation recovery rule, none of them is perfect in the sense that either the fixed point is not strictly followed or the frequency range of self-similar spectra around the fixed point is too narrow. Thus, we are led to the conclusion that the critical 5 -CF superlattices are characterized by two important features: (1) the major peaks cannot be uniquely labeled; (2) the frequency range of self-similarity pattern shrinks to zero.

\section{CONCLUSION}

In this communication, the peak labeling and self-similarity patterns of imaginary wave number spectra are used as essential tools to cross check the nature of $n$-CF superlattices. For $n=3$ and $n=4$-CF superlattices, major peaks can always be labeled uniquely. There exists finite frequency range of self-similarity pattern around the fixed point and the frequency range decreases as $n$ increases; For the critical case of 5-CF superlattices, the unique labeling of major peaks becomes impossible and the frequency range of self-similarity pattern approaches to zero. Our study substantiates the rigorous mathematical proof by $\mathrm{Hu}$ et al. [16], in particular, valuable information is supplemented on the critical 5-CF structures.

\section{Acknowledgments}

This work was supported in part by the National Basic Research Program of China (Grant Nos. 2007CB925104, 2010CB923404). We wish to acknowledge the partial financial support from the NNSFC under Grant Nos. 10774066 and 11021403, and "Excellent Youth Foundation"[10025419].

\section{References}

[1] C. Kittel, Introduction to Solid State Physics (Wiley, New York, 1996), 7th edition. 
[2] E. L. Albuquerque and M. G. Cottam, Phys. Rep. 376, 225 (2003).

[3] D. Shechtman, I. Blech, D. Gratias, and J. W. Cahn, Phys. Rev. Lett. 53, 1951 (1984).

[4] M. Kohmoto, L. P. Kadanoff, and C. Tang, Phys. Rev. Lett. 50, 1870(1983).

[5] S. Ostlund, R. Pandit, D. Rand, H. J. Schellnhuber, and E. D. Siggia, Phys. Rev. Lett. 50, 1873 (1983).

[6] M. Kohmoto, Phys. Rev. Lett. 51, 1198 (1983).

[7] Q. Niu and F. Nori, Phys. Rev. Lett. 57, 2057 (1986).

[8] E. Diez, F. Domínguez-Adame, E. Maciá, and A. Sánchez, Phys. Rev. B 54, 16792 (1996).

[9] R. Merlin, K. Bajema, R. Clarke, F.-Y. Juang, and P. K. Bhattacharya, Phys. Rev. Lett. 55, 1768 (1985).

[10] S. Tamura, and J. P. Wolfe, Phys. Rev. B 36, 3491 (1987).

[11] K. Mizoguchi, K. Matsutani, Shin-ichi Nakashima, T. Dekorsy, H. Kurz, and M. Nakayama, Phys. Rev. B 55, 9336 (1997).

[12] M. Kohmoto, B. Sutherland, and K. Iguchi, Phys. Rev. Lett. 58, 2436 (1987).

[13] W. Gellermann, M. Kohmoto, B. Sutherland, and P. C. Taylor, Phys. Rev. Lett. 72, 633 (1994).

[14] T. Hattori, N. Tsurumachi, S. Kawato, and H. Nakatsuka, Phys. Rev. B 50, 4220 (1994).

[15] E. Maciá, Appl. Phys. Lett. 73, 3330 (1998).

[16] A. Hu, Z. X. Wen, S. S. Jiang, W. T. Tong, R. W. Peng, and D. Feng, Phys. Rev. B. 48, 829 (1993).

[17] V. Elser, Phys. Rev. B. 32, 4892 (1985).

[18] X. H. Yan, J. X. Zhong, J. R. Yan, and J. Q. You, Phys. Rev. B 46, 6071 (1992).

[19] G. Y. Oh, C. S. Ryu, and M. H. Lee, Phys. Rev. B 47, 6122 (1993).

[20] G. Y. Oh and M. H. Lee, Phys. Rev. B 48, 12465 (1993).

[21] X. J. Fu, Y. Y. Liu, Zizheng Guo, Peiqin Zhou, and X. Q. Huang, Phys. Rev. B 51, 3910 (1995).

[22] Peiqing Tong, Phys. Rev. B 53, 1795 (1996). 
[23] G. Y. Oh, H. Y. Choi, and M. H. Lee, Phys. Rev. B 54, 6043 (1996).

[24] S. Thiem, M. Schreiber, and U. Grimm, Phys. Rev. B 80, 214203 (2009).

[25] R. W. Peng, A. Hu, S. S. Jiang, C. S. Zhang and D. Feng, Phys. Rev. B 46, 7816 (1992).

[26] Y. Q. Lu, Y. Y. Zhu, Y. F. Chen, S. N. Zhu, N. B. Ming, and Y. J. Feng, Science 284, 1822(1999).

[27] Weiyi Zhang, Z. X. Liu, and Z. L. Wang, Phys. Rev. B 71, 195114 (2005).

[28] Z. X. Liu and Weiyi Zhang, Phys. Rev. B 72, 134304 (2005).

[29] M. Kohmoto and Y. Oono, Phys. Lett. 102A, 145 (1984).

[30] M. Kohmoto, Bill Sutherland, and C. Tang, Phys. Rev. B 35, 1020(1987). 
Figure 1: The components and piezoelectric superlattice of $n$-CF sequence. (a) All components have an identically positively polarized domain $\left(\bar{L}_{+} \equiv \pi\right)$ plus a negatively polarized one with varying length. (b) An example of $n$-CF superlattice.

Figure 2: The imaginary wave numbers as functions of frequency for 3-CF superlattices. The three components are $\bar{L}_{-}^{1}=\pi, \bar{L}_{-}^{2}=1 \pi / 3$, and $\bar{L}_{-}^{3}=2 \pi / 3$. (a) For 9 th generation; (b) For 12th generation; and (c) For 16th generation. For superlattice of large generation, major peaks can be labeled according to the projection and cut method.

Figure 3: The self-similarity behavior around the fixed point of the cluster center in 3-CF superlattices. (a) For 9th generation; (b) For 16th generation, enlarged around $\bar{\omega}=3$ and scaled by a factor $\left(\lambda_{0}^{(3)}\right)^{7}$. The other parameter settings are the same as Fig. 2.

Figure 4: The indexing of major peaks of imaginary wave numbers. (a) For 4-CF. The four components are: $\bar{L}_{-}^{1}=4 \pi / 3, \bar{L}_{-}^{2}=1 \pi / 3, \bar{L}_{-}^{3}=2 \pi / 3, \bar{L}_{-}^{4}=3 \pi / 3$; (b) For 5 -CF. The five components are: $\bar{L}_{-}^{1}=6 \pi / 3, \bar{L}_{-}^{2}=1 \pi / 3, \bar{L}_{-}^{3}=2 \pi / 3, \bar{L}_{-}^{4}=4 \pi / 3, \bar{L}_{-}^{5}=5 \pi / 3$. For the critical case $n=5$, the peak labeling is not unique anymore.

Figure 5: The self-similarity behavior around the fixed point of the cluster center in 4-CF superlattices. (a) For 12th generation; (b) For 27th generation, enlarged around $\bar{\omega}=3$ and scaled by a factor $\left(\lambda_{0}^{(4)}\right)^{15}$. The other parameter settings are the same as Fig. $4 \mathrm{a}$. 
This figure "Figure-1.png" is available in "png" format from: http://arxiv.org/ps/1012.0171v3 
Fig.2 Da Liu and Weiyi Zhang
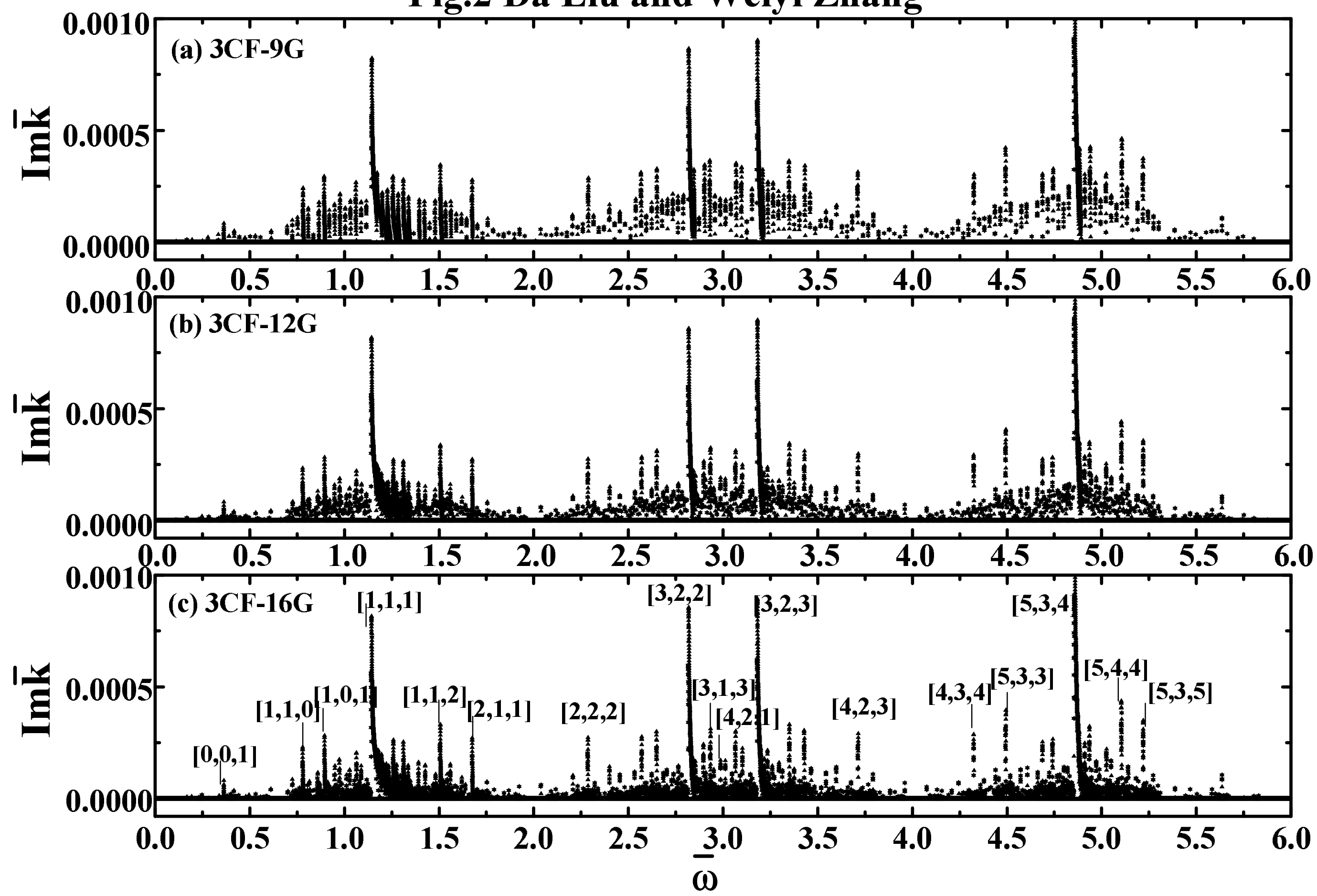
Fig.3 Da Liu and Weiyi Zhang

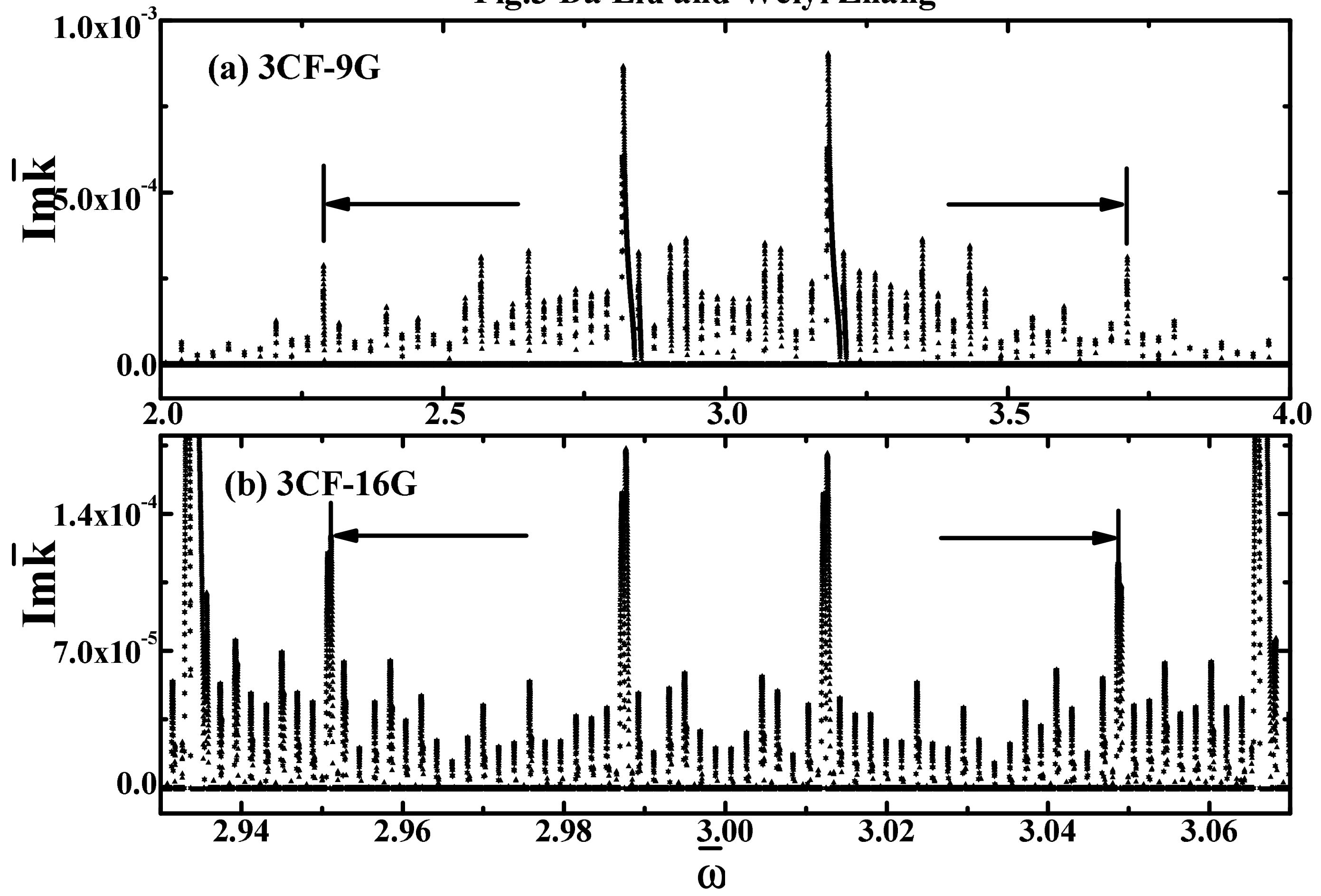


Fig.4 Da Liu and Weiyi Zhang

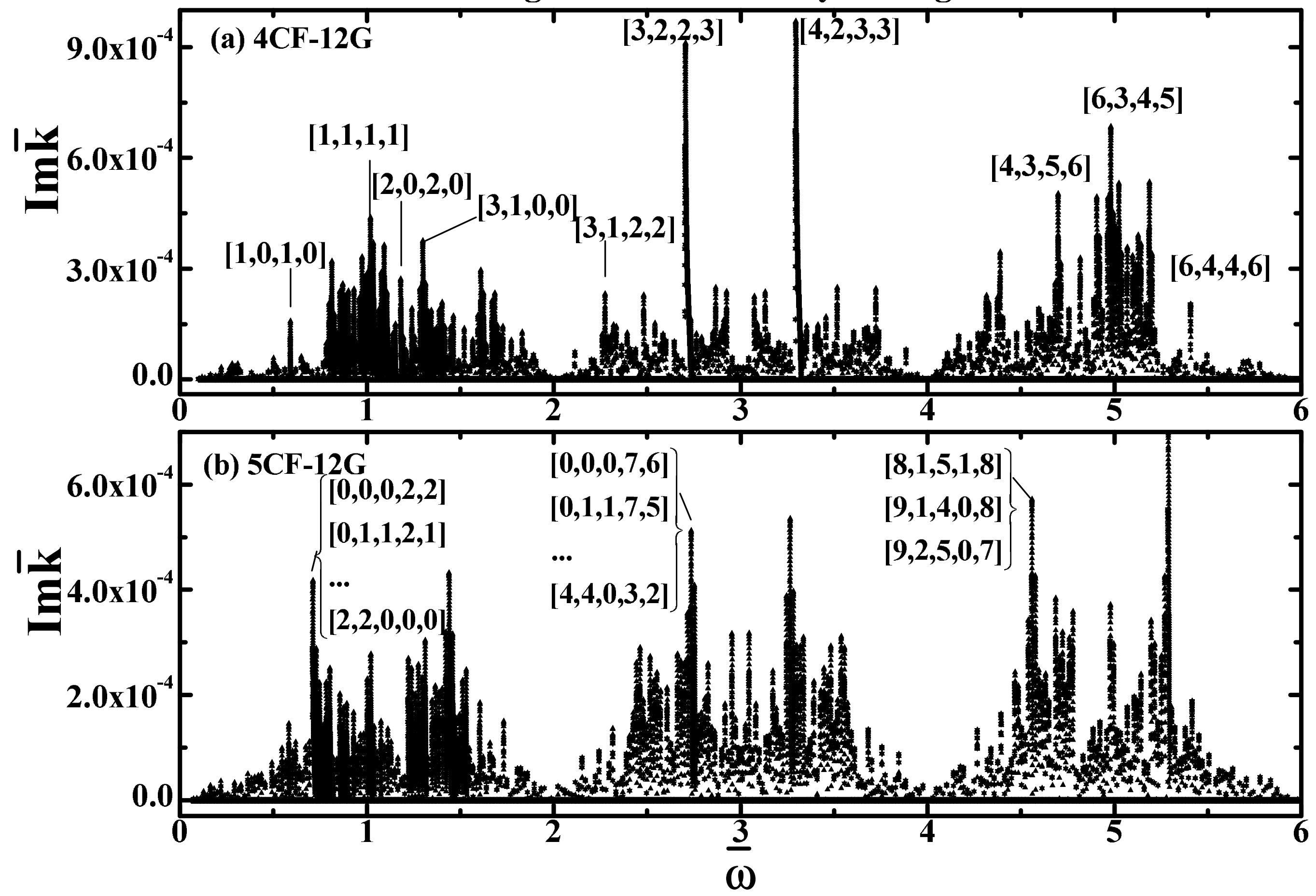


Fig.5 Da Liu and Weiyi Zhang

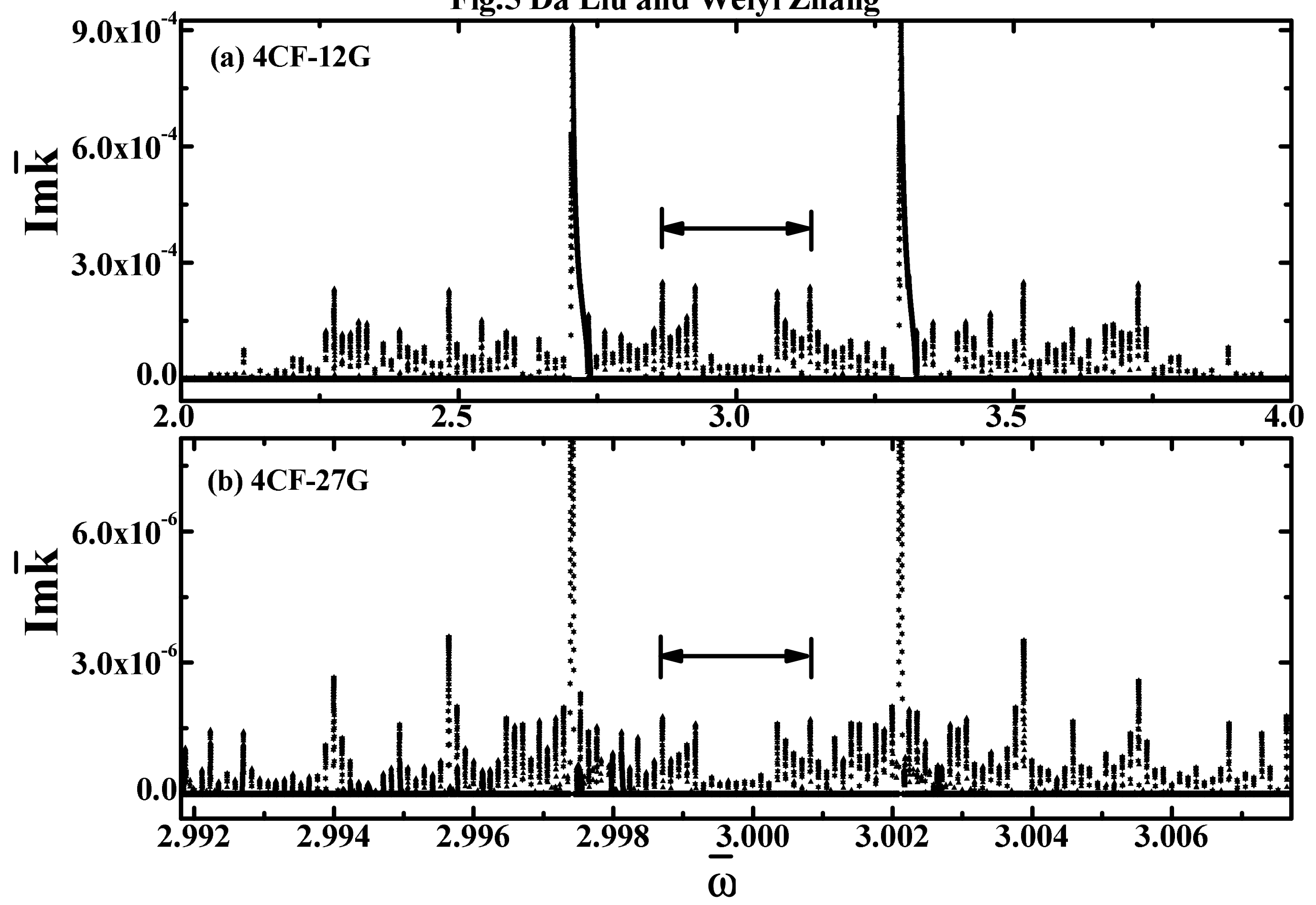

